\title{
Dynamic Design of Text and Exploration of Layout Space
}

\author{
Zhixiong Huang ${ }^{1}$ Long $\mathrm{Li}^{1, *}$ \\ ${ }^{1}$ Fuzhou University, Xiamen, Fujian 361021, China \\ *Corresponding author. Email: 1357326944@qq.com
}

\begin{abstract}
The iteration and development of digital media provides application media for dynamic visual effects, and also enables dynamic visual graphics to be presented. This article attempts to analyze and summarize the current frontier dynamic fonts, construct the visual expression form of dynamic fonts and layout space design management, and explore new ideas for brand communication.
\end{abstract}

Keywords: Dynamic text design, Layout space, Font design.

\section{INTRODUCTION}

In the era of new media, words are no longer limited to the expression of their original intentions. The redesign of words has moved from the expression of its own meaning to the concept of its own meaning in the shape of the font. The emergence of new media promotes the production of new visual expressions. New media is different from traditional media. It gives more space for visual expression. At the same time, visual expression is no longer limited to static design. Compared with static design, dynamic design can more fully present the meaning it needs to present. As the core of the dissemination of visual information, fonts also appear dynamic font design to express and disseminate information.

The designers can combine brand visual performance with dynamic text to explore new ideas for brand communication. For brand communication, a more novel brand communication vision is conducive to brand communication in the market. As a novel form of visual expression, dynamic font design transforms the font from a two-dimensional plane to a threedimensional space and time to change through the dynamic redesign of the font form, so as to realize multi-level, high-density information output in a limited space, capture the user's visual focus more quickly, and promote the brand to achieve efficient information dissemination in a limited time.

\section{THE VISUAL REPRESENTATION OF DYNAMIC FONTS}

\subsection{Dynamic Font Design Under Physical Movement}

The font gives physical movement and makes the transmission of information more interesting. Compared with traditional static fonts, by superimposing the physical properties on the fonts, the fonts are affected by the force to present different dynamic effects on the timeline, and the overall information transmission is also alive. The superposition of different physical movement methods will also result in different textures. When the font has the influence of gravity, the font will sink, and the attribute of the font collider is given at the same time. When the font sinks to the edge of the layout, it can show the movement characteristics of the collision. At this time, the physical texture of the font is hard. If the effect of elasticity is superimposed, the texture of rubber will appear when it collides with the edge of the layout, and different textures will also form different visual feelings and information biases. Movement can not only guide the viewer's visual focus, but also form a visual rhythm. When the font performs physical movement, the viewer's visual focus will be transferred with the movement of the font, so the physical movement can be used to guide the viewer's visual focus to form an efficient visual rhythm. For example, Rupam Raul superimposes 
the gravity attribute on the font $\mathrm{Be}$ humble, so that the fonts show a downward trend in a fixed space, and have collision properties with each other, so

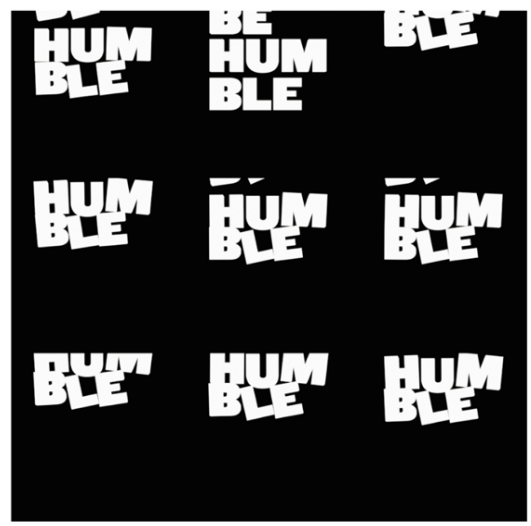

Figure 1 Rupam Raul's dynamic font experiment (1).

\subsection{Dynamic Font Design in Attached Form}

The geometric structure has structural advantages, it can be more tension on the screen, and can produce rich changes at the same time. In common cubes, cylinders, prisms, etc., because of their clear polyhedral structure, the design of dynamic text can be attached to each surface by attaching the text that needs to be presented through the design of attachment, and they can present the information content of different patches on the time axis by wrapping, rotating, zooming, etc., to achieve dynamic text design. that the presentation of dynamic fonts guides the user's vision and strengthens the font experience. ("Figure 1")

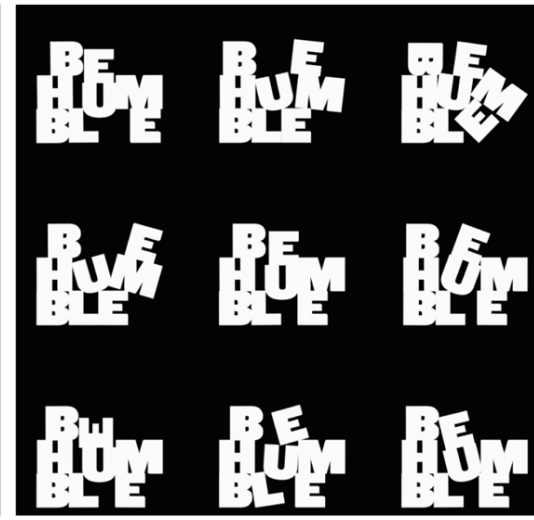

And this way of presentation will produce rich visual changes. It is also possible to repeatedly arrange the presented text on the attached object, and continuously strengthen the text information with the assistance of various physical movements of the object. Rupam Raul's dynamic font experiment, by attaching text to 3D objects of different shapes, and physically moving the 3D objects at the same time, the font also undergoes simple changes. On the one hand, it strengthens the output information, and on the other hand it makes the whole picture more interesting. ("Figure 2")

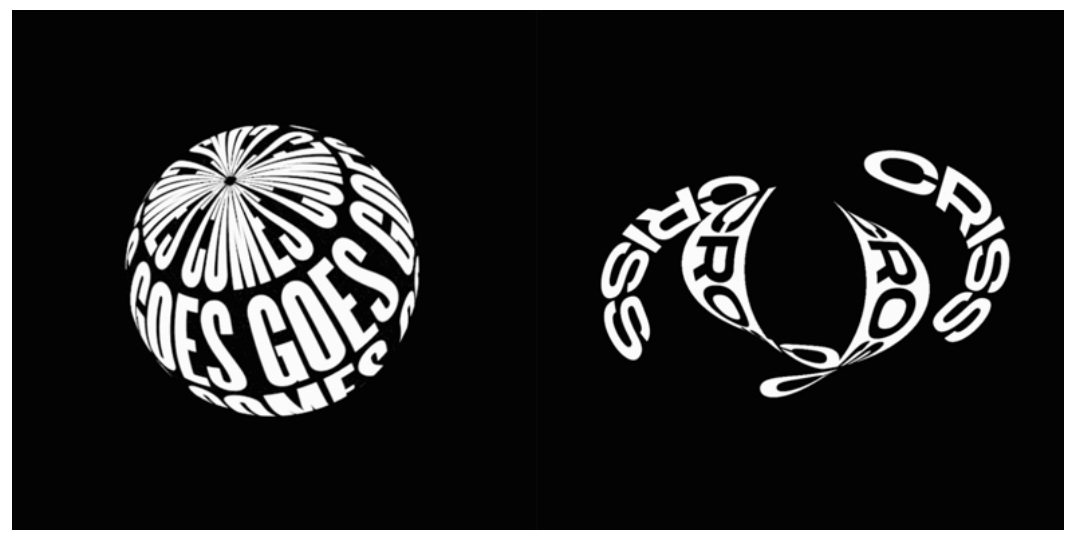

Figure 2 Rupam Raul's dynamic font experiment (2).

\subsection{Skeuomorphic Dynamic Font Design}

People have certain restrictions on the acceptance and cognition of information. At the same time, in real life, it will be affected by objective factors and limit the transmission of information. Therefore, simulating materialization can effectively reduce the cognitive cost of users, so that information can be accepted and recognized by users more quickly. ${ }^{1}$ Skeuomorphic design can quickly reduce the user's acceptance cost and

1. Skeuomorphic interactive interpretation of mobile interactive interface 
cognitive cost by simulating the actual objects, textures, and usage habits that already exist in reality. The user transfers the experience of the existing object to the skeuomorphic design to realize the rapid understanding of the information. The font was originally a pictographic symbol, and gradually became an abstract symbol expression in the subsequent evolution process. Through the skeuomorphic design, the abstract text was reconstructed to simulate the form, use and texture of the representative object, so that users It can quickly realize product cognition, reduce users' thinking, and at the same time, the brand can realize information transmission more efficiently. The rearrangement of multiple fonts to simulate the movement of objects can also achieve skeuomorphic dynamic font design. For example, in the main vision of Shenzhen Fashion Week in 2019, through the redesign of the English initials S, $\mathrm{W}$, and F, the image of the model is used as the main body of visual expression, and the movement of the model is used to form a dynamic text design with rhythmic motion. Through repeated and continuous visual presentations in time and space, users can quickly recognize and convey the brand attitude in a more intuitive way. ("Figure 3")

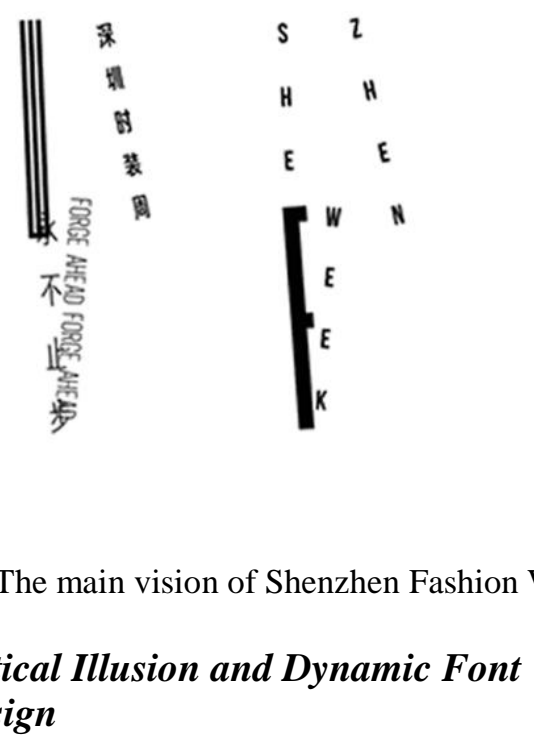

Through the cognitive system, under the influence of environmental conditions, past experience and personal attitudes, when people conduct cognitive activities on objective things, it is easy to cause the production of optical illusions. People usually call the illusion related to the shape, color, space, etc. of an object an optical illusion. [1] Optical illusion can be divided into color optical illusion, geometric optical illusion and so on according to its different manifestations. The optical illusion change of dynamic text is different from the optical illusion change on the twodimensional level. The optical illusion change on the two-dimensional level is the arrangement and combination of the figures, colors, and forms on the two-dimensional picture in accordance with the human visual phenomenon, thereby generating a plane dynamic optical illusion phenomenon. The optical illusion effect of dynamic text is based on the changes of colors, graphics and shapes in a three-dimensional space to form a dynamic optical illusion effect. Through the dynamic manifestation of optical illusion, a more attractive visual hierarchy is formed, and a coherent visual cues and psychological cues are formed for the conversion and output of information on the time axis. The main visual poster of the 2020 Graduation Design Exhibition of Guangzhou Academy of Fine Arts combines GA and FA through the design of GAFA letters, forming an optical illusion through the rendering of transparent materials and the dynamic expression of dynamic rotation. ("Figure 4") 

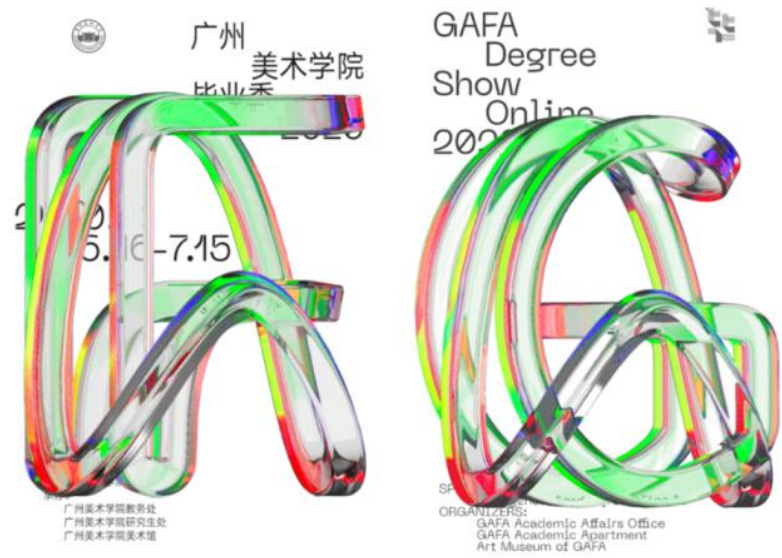

Figure 4 Visual vision of the 2020 Graduation design exhibition of Guangzhou Academy of Fine Arts.

\subsection{Interactive and Dynamic Font Design}

The essence of interaction is to establish the link between the senses and the material, to establish the connection between people and the brand, so that they can influence and interact with each other. With the intervention of the current new media, the design of dynamic fonts can realize the interaction with users, transform the previous single information output carrier into a two-way communication medium with users, link users and brands, and also establish a deeper interactive relationship than ever before. [2] Text content is used as a way for the brand to output information, and the channel for users to receive information is to present dynamic text in an interactive form, which can fully express the information that the text needs to convey, and can also satisfy the user experience to the greatest extent to help brand communication.

\section{DYNAMIC TEXT AND LAYOUT SPACE MANAGEMENT}

\subsection{The Subjectivity of the Layout Space}

In traditional static layout design, information is presented on the layout in a static manner, and the information is conveyed and guided through text, graphics, and colors. The viewer can read the corresponding information according to the guidance. Dynamic layout design has time and space dimensions, as well as movement characteristics, and the amount of information it carries far exceeds that of traditional layout design. It can present multi-dimensional design layout information. Because it appears in a dynamic form, the information conversion speed is faster, which increases the difficulty for the audience to obtain information. [3] While information is changing rapidly, it is difficult for viewers to obtain information quickly, which also makes viewers feel uncomfortable. Therefore, subjectivity is one of the principles that need to be followed in the design of dynamic text layout space. Designers need to take subjectivity as the first element to check the screen, and effectively transmit the subject information through rational and logical analysis and processing.

Subjectivity requires that in dynamic font layout design, the carrier with core information is in the main position in the layout space. Whether it is the core information font itself or the carrier of the core information, it should be at the core position in the layout design, occupying an absolute proportion, so that the viewer can accurately obtain the transmission of the brand's core information when it is first viewed, but will not be disturbed by other elements. Secondly, subjectivity requires continuity in the way of movement with core information carriers. From the perspective of the viewer, a smooth dynamic font design will make the transmission of information very smooth for the viewer. However, if the picture is lagging, it will cause a big obstacle for the viewer to obtain information.

\subsection{Uniqueness of Layout Space}

The various elements in the layout space are not expressed independently, but are designed and combined in a unified manner to form a whole for presentation. The elements in the layout space should interact and complement each other to form a whole. When designing the layout of dynamic 
fonts, a unique element must be formed. The unique element here is composed of fonts, font carriers, and colors.

The unique font starts from a two-dimensional, static point of view, and considers the core information it needs to present, and manages the layout space at the two-dimensional level, so that the viewer can quickly identify it. The unique font carrier should be from a three-dimensional and dynamic perspective. Considering its movement and display in a three-dimensional space, the unique font carrier is more conducive to the reading of information, presenting a clear main body, avoiding fragmented layout, lack of overall sense, and unobtrusive theme. Color is an important element of the layout space, which has a strong impact on the viewer. Statistics people's attention to color accounts for $80 \%$, and the unique color starts from the whole. Due to the motion properties of dynamic fonts, various colors and dynamic effects will make the picture complicated and weaken the readability of the information. Therefore, choosing a unique color can reduce the impact of color on human vision and enhance readability. At the same time, when choosing a color, the color should be consistent with the brand, so as to strengthen the connection between the brand and the viewer and arouse the emotional resonance of the audience.

\subsection{Repeatability of Layout Space}

In the layout design, if it is for the partial design effect in the layout space but ignores the coordination of the overall picture, the final work is unsuccessful. The repetition of a unique element can enhance the effect of the layout, and the transmission of information can also present a complementary state, so that the element can better serve the content.

The repeatability of the layout space is based on the three-dimensional space. In the threedimensional space, the unique elements are combined and arranged through repetitive techniques. The dynamic arrangement can balance the layout and present a variety of visual effects of the layout. The repeated elements can not only play a role in assisting the picture and creating an atmosphere, but also master the equilibrium state of the layout. [4] Therefore, through the repetition in the layout space, the layout effect can be enriched, and at the same time, the information and content that the brand needs to express can be strengthened.

\subsection{Relationship Between Layout Space and Brand}

Brand dynamic design is derived from the extension of the brand design concept based on dynamic graphic design. In fact, it is based on dynamic graphic design, narrowing the scope of its design. It is developed from a graphic brand design system, a dynamic design in which various brand elements are related to each other. As a way of brand dynamic presentation, dynamic text must consider the visual elements of the brand when presented in the layout space. [5] In design, design elements should be taken from the brand design system and dynamically presented in the layout space to prevent the dynamic design from breaking away from the brand and forming an independent visual experience, separating the visual experience of the brand from the dynamic design.

Brands should plan a fixed and unified layout space to present to users. Different from static layout space, because dynamic layout space is in dynamic change on the timeline, the management of layout space needs to consider the proportional relationship between static and dynamic elements. When dynamic elements are more complex and more information is presented, the corresponding static elements should be reduced to prevent the information output of visual elements from appearing to compete with each other. Through the brand's reasonable planning of the layout space, a more orderly visual experience and information output can be realized, and the user experience can be improved.

\section{CONCLUSION}

Under the new media environment and changes in communication media, dynamic visual effects have exerted great communication advantages in brand building. Dynamic graphics have a visual form with a strong overall perception, and at the same time achieve a higher density of information output in a limited layout space, promote the effective dissemination of information, and have a more pleasant visual experience for users. [6] The dynamic font also embodies the vitality of the brand and activates the connection between the brand and the audience. Brand users and evaluators are consumers of the brand. Through dynamic fonts, the brand and consumers are connected and the connection is made alive. Then the connection between the brand and the audience will be closer, 
and the brand will also have the vitality of the brand's own communication.

\section{REFERENCES}

[1] Guan Liwen, Li Dongning, On the Causes and Application Types of Optical Illusion in Dynamic Graphic Design [J]. Modern Decoration (Theory), 2016(12): 101-102. (in Chinese)

[2] Tian Pingping, Analysis on the Interactivity of Font Design in the New Media Environment [J]. Home Drama, 2019, No.316 (16): 131-132. (in Chinese)

[3] Li Lin, Research on Dynamic Poster Layout Design in New Media Environment [D]. North China University of Science and Technology, 2019. (in Chinese)

[4] Wang Jing, Quiet, Extreme, and Active Research on the Dynamic Design of Arrangement in the Internet Era [D]. Zhejiang A \& F University, 2018. (in Chinese)

[5] Li Zhennan, Research on Brand Dynamic Design and Communication under the Background of Mobile Internet [D]. Zhengzhou University of Light Industry, 2020. (in Chinese)

[6] Jia Yongjiao, Exploring the Advantages of Dynamic Graphics Visual Communication in the New Media Era [J]. Journal of News Research, 2020, 11(11): 121-122. (in Chinese) 